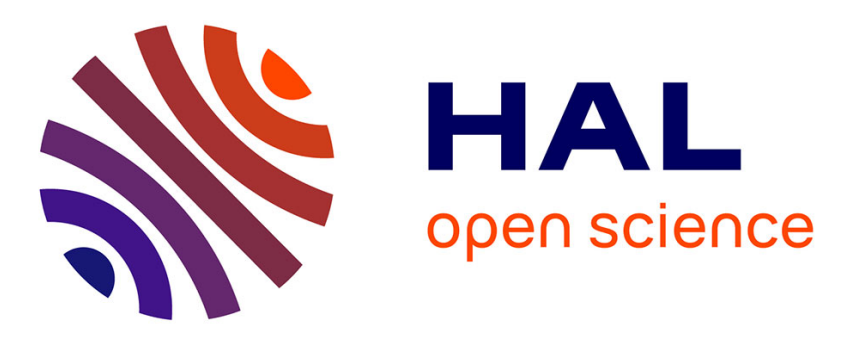

\title{
Narrow Aperture Detection Electrodes ICR Cell with Quadrupolar Ion Detection for FT-ICR MS at the Cyclotron Frequency
}

Konstantin Nagornov, Anton Kozhinov, Edith Nicol, Oleg Yu. Tsybin, David Touboul, Alain Brunelle, Yury Tsybin

\section{To cite this version:}

Konstantin Nagornov, Anton Kozhinov, Edith Nicol, Oleg Yu. Tsybin, David Touboul, et al.. Narrow Aperture Detection Electrodes ICR Cell with Quadrupolar Ion Detection for FT-ICR MS at the Cyclotron Frequency. Journal of The American Society for Mass Spectrometry, 2020, 31 (11), pp.22582269. 10.1021/jasms.0c00221 . hal-02987905

\section{HAL Id: hal-02987905 https://hal.science/hal-02987905}

Submitted on 5 Oct 2021

HAL is a multi-disciplinary open access archive for the deposit and dissemination of scientific research documents, whether they are published or not. The documents may come from teaching and research institutions in France or abroad, or from public or private research centers.
L'archive ouverte pluridisciplinaire HAL, est destinée au dépôt et à la diffusion de documents scientifiques de niveau recherche, publiés ou non, émanant des établissements d'enseignement et de recherche français ou étrangers, des laboratoires publics ou privés. 


\section{A narrow aperture detection electrodes ICR cell with quadrupolar ion detection for FT-ICR MS at the cyclotron frequency}

Konstantin O. Nagornov, ${ }^{1}$ Anton N. Kozhinov, ${ }^{1}$ Edith Nicol, ${ }^{2}$ Oleg Yu. Tsybin, ${ }^{3}$ David Touboul, ${ }^{4}$ Alain Brunelle,, 45 and Yury O. Tsybin ${ }^{1 *}$

${ }^{1}$ Spectroswiss, EPFL Innovation Park, 1015 Lausanne, Switzerland ${ }^{2}$ Laboratoire de Chimie Moléculaire, CNRS UMR 9168, Ecole Polytechnique, Institut Polytechnique de Paris, 91128, Palaiseau, France

${ }^{3}$ Peter the Great Saint Petersburg Polytechnic University, 195251 St. Petersburg, Russia

${ }^{4}$ Université Paris-Saclay, CNRS, Institut de Chimie des Substances Naturelles, UPR 2301, 91198, Gif-sur-Yvette, France

5Laboratoire d'Archéologie Moléculaire et Structurale, LAMS UMR8220, CNRS, Sorbonne Université, 4 place Jussieu, 75005 Paris, France

Correspondence should be addressed to Dr. Yury O. Tsybin, Spectroswiss, EPFL Innovation Park, 1015 Lausanne, Switzerland. E-mail: tsybin@spectroswiss.ch

Running title: 2xNADEL ICR Cell For FT-ICR MS at Cyclotron Frequency 


\section{Abstract}

Ion signal detection at the true (unperturbed) cyclotron frequency instead of the conventional reduced cyclotron frequency has remained a formidable challenge since the inception of Fourier transform ion cyclotron resonance mass spectrometry (FT-ICR MS). Recently, routine FT-ICR MS at the true cyclotron frequency has become a reality with the implementation of ICR cells with narrow aperture detection electrodes (NADEL). Here, we describe the development and implementation of the next generation of these cells, namely a 2xNADEL ICR cell, which comprises four flat detect and four 45 degrees cylindrical excite electrodes, enabling independent ion excitation and quadrupolar ion detection. The performance of the 2xNADEL ICR cell was evaluated on two commercial FT-ICR MS platforms, 10 T LTQ FT from Thermo Scientific and 9.4 T SolariX XR from Bruker Daltonics. The cells provided accurate mass measurements in the analyses of singly and multiply charged peptides (root mean square, RMS, mass error $\Delta m / m$ of $30 \mathrm{ppb}$ ), proteins $(\Delta m / m=200 \mathrm{ppb})$, and petroleum fractions $(\Delta m / m<200 \mathrm{ppb})$. Due to the reduced influence of measured frequency on the space charge and external (trapping) electric fields, the 2xNADEL ICR cells exhibited stable performance in a wide range of trapping potentials (1-20 V). Similarly, in a 13-hour rat brain MALDI imaging experiment, the RMS mass error did not exceed $600 \mathrm{ppb}$ even for low signal-to-noise ratio analyte peaks. Notably, the same set of calibration constants was applicable to Fourier spectra in all pixels, reducing the need for recalibration at the individual pixel level. Overall, these results support further experimental development and fundamentals investigation of this promising technology. 
Keywords: Fourier transform; FT; Fourier transform mass spectrometry; FTMS; ion cyclotron resonance; ICR; quadrupolar ion detection; cyclotron frequency; magnetron frequency; reduced cyclotron frequency; narrow aperture detection electrode; NADEL; 


\section{Introduction}

Fourier transform ion cyclotron resonance mass spectrometry (FT-ICR MS) provides exceptional resolution and mass accuracy performance, given that the number of charges (ions) per measurement cycle (scan) is within a spacecharge limit and does not change significantly scan-to-scan. ${ }^{1-4}$ Indeed, mass spectra of ion populations with a too high number of charges may demonstrate significant deviations in peaks position and abundance and may exhibit artifacts, such as peak coalescence and peak splitting. ${ }^{5-8}$ To accommodate the dependence of FT-ICR MS performance on the electric fields, namely trapping and space charge fields, FT-ICR methods and techniques have been extensively optimized over the past 40 years.9-30 Nevertheless, the fundamental principle of operation for FT-ICR MS remains the detection of ion signals at the reduced cyclotron frequency. That is despite a strong dependence of the reduced cyclotron frequency on the electric fields present in the ICR cell. ${ }^{16}$ On the other hand, FT-ICR MS theory suggests that measuring ion signals at the true (or unperturbed) ion cyclotron frequency can be more precise, due to a less pronounced (or, theoretically, absent) dependence on these fields. ${ }^{11}$, 31-35 However, until recently, there has been no successful implementation of ICR cells operating at the true cyclotron frequency for analytical applications.

It was a serendipitous finding that, during performance evaluation of a novel ICR cell with dipolar ion excitation and dipolar ion detection performed with the narrow aperture detection electrodes (NADEL), 1xNADEL ICR cell, we observed efficient generation of ion signals at the true cyclotron frequency. ${ }^{23,} 36$ The corresponding peak structures in the newly generated 
mass spectra were found to be represented by the true (unperturbed) cyclotron frequency peaks as the base peaks. ${ }^{37} 36$ The subsequent analytical model development, supported by comprehensive ion motion simulations, has revealed the potential mechanism of this process. ${ }^{38,39}$ Namely, upon ion trapping, excitation, and detection in a 1xNADEL ICR cell, ions are experiencing radially non-linear trapping electric fields, with an electric center of the ICR cell displaced from its geometrical center. The resulting motion of an ion ensemble was found to significantly differ from the conventional one established in the quadratic trapping potential. ${ }^{38,} 39$ In turn, ions having slightly different magnetron (drift) frequencies collectively destroy peaks in mass spectra that contain magnetron frequency components (e.g., reduced cyclotron frequency peaks). ${ }^{38}$ In contrast, the same ions together contribute to peaks produced by the (true) cyclotron frequency. However, to achieve an appropriate quality of mass spectra generated at the true cyclotron frequency in the original 1xNADEL ICR cell, fine-tuning of the cell's parameters was needed. The significant asymmetry of trapping potential distribution realized in the 1xNADEL ICR cell may be responsible for the latter. ${ }^{36,38}$

To overcome this limitation of the 1xNADEL ICR cells, we introduce here the next generation NADEL ICR cell, termed the 2xNADEL ICR cell. This novel ICR cell concept integrates the principles of the original 1xNADEL ICR cell with a trapping potential distribution that is significantly more symmetric regarding the geometric center of the ICR cell. The 2xNADEL ICR cell boosts four cylindrical ion excitation electrodes and four flat ion detection electrodes independent of the excitation electrodes. In addition to the conventional 
dipolar ion detection, the novel arrangement allows performing quadrupolar ion detection. The latter is known to enhance the efficiency of ion signal detection at the (true) cyclotron frequency. ${ }^{38-40}$

The original analytical theory of quadrupolar ion detection favoring ion signal measurement at the cyclotron frequency was formulated and experimentally verified by Schweikhard and co-workers. ${ }^{40}$ Their approach to quadrupolar ion detection implied the use of linear trapping fields (quadratic potential distribution generated by electrodes of a hyperbolic shape) and the compact ion bunch principle of ion excitation and detection. Overall, the experimental results obtained by Schweikhard and co-workers demonstrated the apparent advantages of quadrupolar ion detection for ion signal measurements at the true cyclotron frequency. However, the implemented ICR cells remained limited to serving particular questions of authors' interests in precision mass spectrometry of atomic ions. ${ }^{41}$ Performance-wise, the reported mass (frequency) spectra demonstrated a visible contribution from the second harmonic of the reduced cyclotron frequency.

The described here 2xNADEL ICR cells were implemented in two major types of FT-ICR MS instruments, an LTQ FT instrument from Thermo Scientific and a SolariX XR instrument from Bruker Daltonics, without (additional) hardware or software modifications of the original set-ups. Both implementations delivered an improved performance of FT-ICR MS at the true cyclotron frequency compared to the 1xNADEL ICR cell implementation. Compared with the measurements performed by Schweikhard and coworkers, the 2xNADEL ICR cell implementations reported here demonstrate detection of, almost exclusively, peaks at the true cyclotron frequency, 
whereas peaks at the second harmonic of a reduced cyclotron frequency are absent or minor. Notably, the 2xNADEL ICR cells enable mass measurements of (bio)molecules in a wide range of masses and charge states, supporting modern applications of FT-ICR MS in protein analysis, imaging, and petroleomics.

\section{Experimental methods}

Sample preparation. MRFA, substance $\mathrm{P}$, and equine myoglobin were obtained from Sigma-Aldrich (Buchs, Switzerland). LC-MS grade acetonitrile and water, as well as phosphoric acid and trifluoroacetic acid (TFA) were obtained from Fluka (Buchs, Switzerland). Peptides P1, P2, and P3 with the following sequences: P1 - EESTR, P2 - PMMSR, and P3 - HDGHR were synthesized inhouse with solid-state peptide synthesis. Formic acid was obtained from Merck (Zug, Switzerland). Peptide and protein solutions were prepared in 1:1 $(\mathrm{v} / \mathrm{v})$ water/acetonitrile solvent mixtures containing $0.1 \%(\mathrm{v} / \mathrm{v})$ of formic acid. The final peptide and protein solution concentrations were $\sim 2 \mu \mathrm{M}$.

Tissue sections for MS imaging experiments of $10 \mu \mathrm{m}$ thickness were cut from fresh frozen healthy rat brain using a Leica CM3050 cryostat (Leica Microsystems $\mathrm{GmbH}$, Wetzlar, Germany) at $-20^{\circ} \mathrm{C}$. These sections were thawmounted onto ITO-coated glass microscope slides and stored at $-80{ }^{\circ} \mathrm{C}$ until further processing. Before analysis, tissue sections were put under vacuum at a pressure of a few $\mathrm{hPa}$ for $10 \mathrm{~min}$ to eliminate water from tissue sections. They were then coated with an a-cyano-4-hydroxycynamic acid matrix, purchased from Sigma-Aldrich (Saint-Quentin-Fallavier, France). Matrix 
solution was prepared in acetonitrile/water/TFA $(70 / 30 / 0.1, \mathrm{v} / \mathrm{v} / \mathrm{v})$ at a concentration of $10 \mathrm{mg} / \mathrm{mL}$. The matrix was homogeneously sprayed onto the tissue section with a TM-Sprayer (HTX Imaging, Carrboro, NC, USA). This system is equipped with a nozzle making an aerosol of matrix droplets by moving above the fixed sample. The combination of heat (the nozzle temperature is controlled at $70^{\circ} \mathrm{C}$ ), pressure (carrier solvent at a flow rate of $240 \mu \mathrm{L} / \mathrm{min}$, high pressure by an isocratic pump), and drying sheath gas (dry nitrogen at a pressure of $\sim 700 \mathrm{hPa}$ ) results in a precisely-controlled and reproducible solvent/matrix deposition. ${ }^{42}$

Mass spectrometry. Experiments were performed using a 9.4 T FT-ICR MS SolariX XR instrument originally equipped with ParaCell (Bruker Daltonics, Bremen, Germany) and a hybrid 10 T LTQ FT-ICR MS initially equipped with the Ultra cell (Thermo Fisher Scientific, Bremen, Germany). The 2xNADEL ICR cells (Spectroswiss, Lausanne, Switzerland) with four narrow aperture detection electrodes and four wide (about 45 degrees each, cylindrical) excitation grid-electrodes were designed and benchmarked using SIMION environment for ion motion simulations, Figure 1 and Figure S1 (Supporting Information). Two different configurations of the 2xNADEL ICR cells were custom manufactured and implemented to enable installation with both rear (Bruker-type) and front (Thermo-type) ICR cell mounting approach, Figure S2 (Supporting Information).

Two pairs of trapping rings were used for ion confinement (trapping), Figure S1. Ion motion was excited using a dipolar broadband RF sweep applied to the adjacent excite grid-electrodes. In contrast, other excite grid-electrodes 
were grounded at all times, Figure 1, bottom right panel. The applied dipolar adjacent excitation scheme allowed for exciting simultaneously both cyclotron and magnetron (drift) ion motions, which is crucial for the establishment of the true cyclotron regime. ${ }^{36}$ Advantageously to alternative implementations, there is no need for external electronics switching between ion detection and ion excitation to realize quadrupolar ion detection with the 2xNADEL ICR cell configuration, Figure 1 top right panel. The ParaCell and Ultra ICR cell were operated using standard dipolar excitation and detection schemes utilizing opposite excitation electrodes.

Time-domain signals (transients) were sampled using commercial built-in data acquisition systems (Bruker Daltonics or Thermo Fisher Scientific, correspondingly). On the LTQ FT instrument, time-domain signals were recorded in MIDAS file format (.dat files) using advanced user interface capabilities (Thermo Fisher Scientific). On the SolariX XR instrument, timedomain signals (fid/ser files) were acquired following common strategies and stored in d. folders. Experimental sequences for both instruments were controlled with the standard electronics and software. Processing of transients acquired with both systems (Thermo Fisher Scientific and Bruker Daltonics) and comprehensive data analysis of the resulting mass spectra were performed using Peak-by-Peak software (Spectroswiss). Briefly, selected individual transients or the sum of several individual transients were zerofilled twice unless stated otherwise, apodized with the Kaiser (full or half) window, and Fourier transformed in magnitude (mFT) or absorption (aFT) mode. Absorption-mode processing of transients in MIDAS .dat files (Thermo Fisher Scientific) and .d folders with with fid/ser files (Bruker Daltonics) was 
enabled by the use of AutoVectis software (Spectroswiss). ${ }^{43}$ Peak picking was performed using three-point parabolic interpolation. Peaks of intensity higher than six sigma of a noise, $6 \sigma$, were considered as analyte signals. Therefore, the signal-to-noise ratio (SNR) of one equals to $6 \sigma$. The sigma values were calculated using a logarithmic noise thresholding algorithm considering features of mFT and aFT frequency (mass) spectra, as described elsewhere. ${ }^{44}$

Experimental sequence on the LTQ FT instrument. Following the standard operating principles of the LTQ FT instrument, the user first sets the desirable number of charges to be injected into the ICR cell, as controlled by the automatic gain control (AGC) function. ${ }^{1}$ Ions are initially localized within an external linear ion trap, LTQ, and accumulated during the pre-calculated time required to reach the preset AGC value. The preset number of charges (AGC value) was varied from $1 \mathrm{e} 4$ to $5 \mathrm{e} 6$. Further, ions were ejected from the LTQ, trapped in an ICR cell via gated trapping, and excited with a non-adjustable trapping potential of $3 \mathrm{~V}$ at all times for both the Ultra ICR cell and the 2xNADEL ICR cell. A dipolar broadband frequency-sweep excitation (varied from $72 \mathrm{kHz}$ to $720 \mathrm{kHz}$ at $150 \mathrm{~Hz} / \mu \mathrm{s})$ with a peak-to-peak amplitude $V_{\text {exc }}$ in the range of $0-100 \mathrm{~V}$ was applied for a fixed duration of $20 \mathrm{~ms}$ to excite ions to a detectable radius. For the ion detection event, the trapping potential was varied between $0-10 \mathrm{~V}$.

Standard differential dipolar ion detection for the Ultra ICR cell and quadrupolar ion detection for the 2xNADEL ICR cell were used to acquire analyte ion signals. Data sampling frequency was $1.4,2.7$, or $5.5 \mathrm{MHz}$, whereas acquisition time was varied between $96 \mathrm{~ms}$ and $25 \mathrm{~s}$. Acquisition of 
long, up to $25 \mathrm{~s}$, transients was achieved by use of advanced user interface capabilities (Thermo Fisher Scientific). Additionally, intensities of sidebands and peak shapes were optimized via independent variation of four DC offset potentials: one pair of offset potentials was applied to two non-grounded excitation electrodes, and the other pair to two pairs of interconnected flat detection electrodes, Figure 1, throughout the entire experimental sequence. In contrast to the $1 \times$ NADEL ICR cell operation, DC offset potentials applied to the flat detection electrodes did not exhibit a pronounced tuning effect for the 2XNADEL ICR cell with a quadrupolar ion detection scheme as the opposite electrodes were interconnected. The DC offset potentials were set to values from $-100 \mathrm{mV}$ to $100 \mathrm{mV}$ with a step of $1 \mathrm{mV}$ for the positive and negative ion modes independently.

Experimental sequence on the SolariX XR instrument. Experimental sequence employed for the measurements on the SolariX XR instrument was similar to the one on the LTQ FT instrument, except the AGC function (not available). The employed ParaCell was originally installed on this instrument in May 2014 and supported only dipolar detection at the "one omega" frequency. In addition to the standard DC bias shimming at the mantle DC electrodes, a complementary software option allowed injection shimming during the injection of ions into the ParaCell. Ions were initially localized within an external collision cell and accumulated during a preset fixed time. Ion accumulation time was varied in the range from 10 to $300 \mathrm{~ms}$. Further, ions ejected from the external collision cell were trapped inside an ICR cell in the high magnetic field region. In the case of ParaCell, a standard trapping mode 
with a sidekick offset of $-1.5 \mathrm{~V}$ was employed. The 2xNADEL ICR cell was operated using a gated trapping mode with a voltage ramp from $3.0 \mathrm{~V}$ to $5.0-$ 8.0 V (the same trapping potential was applied to both front and back trapping rings) of $20 \mathrm{~ms}$ duration with a sidekick offset potential of $0.0 \mathrm{~V}$. The trapping potential during ion excitation and detection was varied between 0 and $25 \mathrm{~V}$. A dipolar broadband decreasing frequency-sweep excitation (in a variable range of frequencies depending on the preset mass range) with a power of 8 $10 \mathrm{~dB}$ was applied for an adjustable period of $10-20 \mathrm{~ms}$ to excite ions to a detectable radius.

Standard differential dipolar ion detection for ParaCell and quadrupolar ion detection for the 2xNADEL ICR cell were employed to acquire analyte ion signals. Time-domain data sampling frequency was $1.4 \mathrm{MHz}$ for broadband and $20 \mathrm{kHz}$ for narrowband mass measurements, acquisition time (transient length) was varied between $100 \mathrm{~ms}$ and $100 \mathrm{~s}$. Intensities of sidebands and peak shapes were optimized via independent variation of four DC offset potentials throughout the entire experimental sequence in the same way as on the LTQ FT instrument as described above. ${ }^{45}$ The DC potentials were set to values between $-1.5 \mathrm{~V}$ and $1.5 \mathrm{~V}$ with a step of $1 \mathrm{mV}$ for the positive and the negative ion modes independently. Matrix-assisted laser desorption ionization (MALDI) imaging experiments were performed with a laser frequency of $2 \mathrm{kHz}$ and power of 22 units; 80 laser shots per pixel with a $50 \mu \mathrm{m}$ pixel size. 


\section{Results and Discussion}

Design and implementation of the 2xNADEL ICR cells. The overall concept of the implemented 2xNADEL ICR cells follows the original design of the Ultra ICR (Thermo Fisher Scientific) and 1xNADEL ICR cells, Figure 1 and Figures S1 and S2, Supporting Information. ${ }^{1,23}$ The associated features include: (i) application of the excite RF field to the grids positioned inside the ICR cell and extending beyond the detect electrodes, ensuring an appropriate (uniform) excitation field distribution along the whole length of the detect electrodes; and (ii) possibility to apply DC offset potentials to excite and detect electrodes. The principal difference in the designs of the 2xNADEL ICR cell and the original 1xNADEL ICR cell is the additional pair of narrow aperture detection electrodes accommodated by the 2xNADEL ICR cell, Figure 1. These flat detection electrodes are of a similar shape, and they are inserted radially inwards at the same distance, as in the previously described 1xNADEL ICR cell. ${ }^{23}$ Increasing the number of detection electrodes from two to four results in the following changes in the overall cell properties: (i) distribution of the trapping electric field remains radially non-linear, but it is now symmetric about the geometric center of the cell (a squircle, or super-ellipse, configuration of equipotential curves), Figure 1 bottom left panel; (ii) number of excite electrodes is doubled, creating a possibility to perform ion excitation not only via standard dipolar (or even quadrupolar or quadrature) excitation by the use of the opposite excite grid-electrodes, but also by applying the adjacent electrode excitation scheme, Figure 1 bottom right panel; and (iii) quadrupolar (and quadrature) ion detection can now be enabled.46 
Conveniently, the employed detection scheme does not require an electronic switch between excite and detect electrodes for alternating between ion excitation and detection processes, Figure 1 top right panel and Figure S1, Supporting Information. The latter is beneficial because an external circuitry is eliminated and potential signal artifacts are thus not introduced. The fundamentals of ion motion established in the NADEL ICR cells for ion detection at the true cyclotron frequency, schematically shown in Figure 1 top right panel for the case of ion slab formation (when cyclotron and magnetron radii after ion excitation are equal), have been described elsewhere. ${ }^{38,} 39$

The 2xNADEL ICR cells, as described in Figure 1, were constructed and fitted onto two ion optics configurations enabling cell installation at either the LTQ FT-ICR MS from Thermo Scientific or SolariX XR FT-ICR MS from Bruker Daltonics (Figure S2, Supporting Information). The principal difference between these designs is the following: ICR cell in the Thermo instrument is fitted to the end of the ion transfer multipole, ensuring that ion transfer optics is aligned with the ICR cell and ions enter the ICR cell on-axis; ${ }^{1}$ whereas the ICR cell in the Bruker instrument is fitted to the back flange of the vacuum tube and has no physical connection to the ion transfer optics employed for ion injection into the ICR cell. ${ }^{47}$ Owing to the capabilities of both LTQ FT and SolariX XR FT-ICR MS instruments employed, all the required electric signals were readily provided, and no (additional) hardware modifications were needed to perform test measurements with the 2xNADEL ICR cells.

Initial characterization of the $2 x N A D E L$ ICR cell. One of the principal properties of a peak detected at the true cyclotron frequency is a reduced dependence of 
peak position (frequency value) on a change in the trapping potential compared to the properties of the reduced cyclotron frequency peak. ${ }^{36}$ Indeed, the peak position in a frequency domain is known to follow the direct dependence on the trapping potential when reduced cyclotron frequency is measured. The higher the trapping field - the lower is the reduced cyclotron frequency because of the linear dependence of the magnetron (drift) frequency on the trapping potential. ${ }^{48}$ In the case of ion signal detection at the true cyclotron frequency, magnetron frequency is not a part of an equation and, thus, peak position in the frequency domain should not depend on the trapping potential.11 Figure 2 confirms that the 2xNADEL ICR cell with quadrupolar ion detection enables true cyclotron frequency measurements that are practically independent of the trapping potential in the whole range of trapping potentials available on a SolariX XR instrument. A similar behavior was obtained for the LTQ FT set-up for the range (which is more narrow than that of SolariX XR) of trapping potentials supported by this instrument (Figure S3, Supporting Information). Compared to the earlier reported peak position dependence on trapping potential for dipolar ion detection realized in the 1xNADEL ICR cell, 36 results reported here indicate superior performance of the 2xNADEL ICR cell with quadrupolar ion detection for cyclotron frequency measurements. The latter is reflected through a reduced variation of the peak frequency with respect to the trapping potential, practically absent reduced cyclotron frequency peak, and easier tuning of the ICR cell parameters to achieve the best performance. For example, with a singly charged MRFA monoisotopic ion, the peak at the true cyclotron frequency can be substantially, up to three orders of magnitude, higher in SNR than peaks at 
the first and second harmonics of the reduced cyclotron frequency (Figure S4, Supporting Information).

In comparison with ICR cells employed in FT-ICR MS up to date, the ability to support measurements at trapping potentials above $15 \mathrm{~V}$ can be considered as unique. Indeed, until the development and implementation of the dynamically harmonized cell (DHC, or ParaCell) and its improved versions, ${ }^{49}$ ion detection took place usually at trapping potentials not exceeding 1-2 V.1, 9, 19 The DHC (or ParaCells) enabled routine operation also at higher trapping potentials of up to 3-6 V, and, potentially, even higher ones. ${ }^{14}$ Indeed, Figure 2 shows an expected dependence of peak position as a function of trapping potential for ion detection with a conventional ParaCell on a 9.4 T SolariX XR FT-ICR MS. Increased trapping potential results in the reduction of the measured frequency. What is more striking is the dependence of the SNR of the ion signal as a function of trapping potential for both ParaCell and 2XNADEL ICR cells installed in the same instrument one after another. Increased trapping potential for detection at the true cyclotron frequency (2xNADEL ICR cell) shows a maximum SNR at around $12 \mathrm{~V}$. In contrast, ion detection at the reduced cyclotron frequency (ParaCell) shows significant ion signal dampening for trapping potentials exceeding $3 \mathrm{~V}$ resulting in a signal loss at about $10 \mathrm{~V}$. Therefore, to the best of our knowledge routine FT-ICR MS applications at trapping potentials of $15-20 \mathrm{~V}$ have not been reported until now. This intriguing performance of the 2xNADEL ICR cells may create opportunities for improved detection of (energetic) product ions for the in-cell tandem mass spectrometry techniques, such as electron capture dissociation (ECD). 50 The upper limit of sustainable trapping potentials is yet to be 
established experimentally for the 2xNADEL ICR cells. Notably, to obtain the reported ParaCell results, the commercial instrument was operated at the best possible conditions by an experienced operator.

Performance evaluation of the 2xNADEL ICR cell: mass accuracy. Mass accuracy performance of the 2xNADEL ICR cell operating in the true cyclotron frequency regime was estimated via broadband mass spectrometry analysis of a poly-phosphoric acid, poly- $\left(\mathrm{H}_{3} \mathrm{PO}_{4}\right)_{n}$. Single scan broadband mass spectra acquired on both platforms provide comparable performance characteristics, Figure 3. First, these results confirm the overall 2xNADEL ICR cell applicability to mass measurements of molecular mixtures in a broad mass range without noticeable artifacts, e.g., harmonics. Secondly, the corresponding mass error distributions plotted for 11 monoisotopic peaks of poly-phosphoric acid and measured with external calibration in the mass range of $300-1500 \mathrm{~m} / \mathrm{z}$ demonstrate a root-mean-square (RMS) error of 94 ppb for the LTQ FT platform (2174 single scans considered for mass error calculation) and an error of $87 \mathrm{ppb}$ for the SolariX XR platform (87 single scans considered).

Similarly, analysis of TFA performed in the true cyclotron frequency regime on the 10 T LTQ FT yields mass accuracy of 200-300 ppb for 30 peaks in a wide, 300-3000 m/ $\mathrm{z}$, mass range (Figure S5, Supporting Information). The minor but visible asymmetry in the error distributions in Figure 3 and Figure S5 (Supporting Information) may reflect the complex composition of the considered mass spectra, which contain both high and low abundance components. As a result, mass spectra calibration performed on the high 
abundance ions may show deviations for the lower abundance ions. The latter is further enhanced by the overall very high mass accuracy levels reported for these measurements, more typical for petroleomics-type applications, which suggests the need in a more comprehensive recalibration procedure. ${ }^{51}$

Addressing increased sample complexity, as it takes place in FTMS-based petroleomics applications, is another valuable diagnostic test for mass accuracy performance evaluation. The 2 xNADEL ICR cell operating in the true cyclotron frequency regime on the LTQ FT platform demonstrates petroleomics-grade performance detecting about 14,600 compounds with an RMS of $164 \mathrm{ppb}$, Figure 4. Both external and internal recalibration procedures were employed to obtain these results following the previously described approaches. ${ }^{36,51,52}$ Importantly, phase properties of ion signals, even in a complex mixture analysis, allow the application of absorption mode FT (aFT) processing, which is known to increase the achievable resolution in comparison with the magnitude mode FT (mFT) signal processing. ${ }^{43}$, 52 Representing mass spectra in the aFT mode leads to an increase in the number of considered monoisotopic peaks to 14,674 (more than 41,000 peaks in total, 3 s detection period) compared with 34,500 peaks detected in the mFT mass spectra, Figure 4 left panel. Here we benchmark an enhanced performance with the 2xNADEL ICR cell for the previously reported petroleum aFT mass spectrum that was acquired by use of a 1xNADEL ICR cell (true cyclotron frequency via dipolar ion detection). ${ }^{52}$ Further method development, particularly segmented data acquisition and processing, should provide an additional performance boost as demonstrated by Barrow and co-workers, and others. ${ }^{24,53,54}$ Other modern method developments, including the use of on- 
line liquid separation of petroleum-type samples should reveal even more molecular features, as suggested by an approach spearheaded by Rodgers and co-workers. ${ }^{55}$ Therefore, a combination of modern methods should be employed to comprehensively benchmark the 2xNADEL ICR cell at the true cyclotron frequency to evaluate its standing and value in the contemporary petroleomics field.

Another mass accuracy performance evaluation test is a challenge of mass measurements of singly charged species in an imaging FTMS experiment. ${ }^{25-27}$ Indeed, pixel to pixel space charge variation can be substantial, for example, in MALDI-based imaging FTMS (Figure S6, Supporting Information), whereas total ion charge control is not possible. ${ }^{25}$ Therefore, imaging FTMS relies on the space charge capacity of mass analyzer. Conventional reduced cyclotron frequency imaging FTMS workflows succeed to deliver relatively good levels of mass accuracy due to sophisticated, pixel by pixel, data recalibration approaches and use of ultra-high field magnets. ${ }^{25-27}$ Nevertheless, space charge effects are to be taken into account for accurate data analysis even for high magnetic field FT-ICR MS instruments. Performing MALDI imaging FTMS at the true cyclotron frequency provides an intriguing capability to reach excellent mass accuracy performance even with a single set of calibration coefficients for the whole image, Figure 5. Examples shown are the images of three cations, identified as $\left[\mathrm{C}_{40} \mathrm{H}_{80} \mathrm{NO}_{8} \mathrm{P}+\mathrm{K}\right]^{+}, \quad\left[\mathrm{C}_{48} \mathrm{H}_{84} \mathrm{NO}_{8} \mathrm{P}+\mathrm{K}\right]^{+}$, and $\left[\mathrm{C}_{39} \mathrm{H}_{75} \mathrm{O}_{7} \mathrm{P}+\mathrm{K}\right]^{+}$by the on-line metabolite identification tool METASPACE. ${ }^{56}$ The corresponding RMS mass accuracies (errors) across all pixels for these images are in the range of 300-600 pp. The higher abundance compound, $\left[\mathrm{C}_{40} \mathrm{H}_{80} \mathrm{NO}_{8} \mathrm{P}+\mathrm{K}\right]^{+}$, which demonstrates SNR values up to 100 , exhibits an 
almost 2-fold narrower mass error distribution compared to the low abundance compounds, $\left[\mathrm{C}_{48} \mathrm{H}_{84} \mathrm{NO}_{8} \mathrm{P}+\mathrm{K}\right]^{+}$and $\left[\mathrm{C}_{39} \mathrm{H}_{75} \mathrm{O}_{7} \mathrm{P}+\mathrm{K}\right]^{+}$, which are detected with the SNR values below 12. Overall, this is an appropriate result taking into account a moderate magnetic field of $9.4 \mathrm{~T}$ and the use of a single set of calibration coefficients employed for calibration of mass spectra in all pixels. These results further confirm the general applicability and potential benefits of the 2xNADEL ICR cell with true cyclotron frequency detection for imaging applications.

Performance evaluation of the 2XNADEL ICR cell: peptide and protein analysis. Analysis of peptides and proteins generally implies mass measurements of multiply charged species, often with several charge states present in the ICR cell at the same time, raising a question on how these ions would be distributed in the 2xNADEL ICR cell. Our previous work on computer simulations of ion motion suggests a co-existence of these ion distributions. ${ }^{39}$ On the other hand, it also confirms a drastic difference in ion motion characteristics between the standard and described here frequency detection methods.

First, we demonstrate that, for analysis of a doubly charged peptide substance $\mathrm{P}$ at the true cyclotron frequency, Figure S7 (Supporting Information), resolution and SNR as functions of the detection period support results reported in Figure 2. The detected transient signal shows a limited decay over a very long period of time, up to $25 \mathrm{~s}$ (LTQ FT platform). As a result, the SNR values of ion signal continue increasing with the increase in ion detection period for the whole considered range. The afforded transient periods 
enable resolving signals from isobaric peptides, vide infra, which can be useful for the analysis of peptide isotopic fine structures.

Naturally, intact protein analysis is a challenge for ion signal detection at the true cyclotron frequency. Impressively, the 2xNADEL ICR cells demonstrate efficient broadband detection of a protein (myoglobin, 17 kDa) in diverse charge states at the true cyclotron frequency, Figure 6 (9.4 T SolariX XR platform) and Figure S8, Supporting Information (10 T LTQ FT platform). Results from both platforms demonstrate baseline resolved isotopic patterns for any detected protein charge state. Experimental data show that only (or primarily) peaks corresponding to the true cyclotron frequency are present in the mass spectra. Visibly, there are no abundant peaks of the reduced cyclotron frequency or its harmonics in the mass spectra. As discussed in the prior publications on ion motion simulations, reduced cyclotron frequency peaks (at any harmonic) may be present in mass spectra acquired in the true cyclotron frequency regime. ${ }^{38,} 39$ Still, due to their peak decomposition from many ions giving an input, their signals are buried into the noise in the real-life experimental data. ${ }^{38}$

Moreover, appropriate phase properties of ion signals, including stability of the initial phase over the detection period, allow aFT mass spectra representation with increased resolution, Figure 6. The shown example demonstrates up to a 2 -fold increase relative to resolution values according to the FT-ICR instrument specifications, as expected. That is a direct consequence of the employed absorption mode versus magnitude mode FT processing options. Overall, these results indicate that ion detection at the 
true cyclotron frequency has the potential to match performance requirements in modern FTMS applied to peptide and protein analysis.

Performance evaluation of the 2XNADEL ICR cell: space charge effects. To evaluate frequency stability during ion detection and maximum achievable resolving power, we acquired extended period transients of peptides, Figure 7. In the first example, analysis of a triplet of isobaric synthetic peptides demonstrates ion signal coherence over an extended detection period (50 s transients, 9.4 T SolariX XR FT-ICR MS platform), yielding the resolution exceeding five million at $620 \mathrm{~m} / z$ (mFT processing), Figure 7 left panel. Notably, the detected species are baseline resolved despite their very close mass values, with a mass difference corresponding to about the mass of an electron $(0.5 \mathrm{mDa})$. Previously, this mass split was resolved, for example, by applying FT-ICR MS at the reduced cyclotron frequency to a doublet of isobaric species. ${ }^{57}$ The same isobaric peptide triplet was resolved with a $25 \mathrm{~s}$ transient (resolution exceeding three million, mFT processing) on the $10 \mathrm{~T}$ LTQ FT platform equipped with a 1 XNADEL ICR cell operating in the reduced cyclotron frequency regime. ${ }^{23}$ In addition to baseline resoltution of these isobaric species, results shown in Figure 7 left panel demonstrate that averaging of transients from 25 independent measurements (scans) preserves peak shape and resolution. Therefore, any scan-to-scan variation of detected ion frequencies is much less than the achieved peak width and insignificant.

Similarly, mass resolution exceeding 10 million at $650 \mathrm{~m} / z$ was achieved for a singly-charged peptide (transient length $97 \mathrm{~s}$, heterodyne mode, mFT processing, 9.4 T SolariX XR FT-ICR MS platform), Figure 7 right panel. 
Theoretically, the ability to preserve ion motion coherence over these extended periods in the cyclotron frequency regime in the 2xNADEL ICR cell is supported by the spatial dispersion of ions in the cell volume, resulting in reduced space charge-induced transient decay. ${ }^{38,39}$

The relatively low abundance of ion signals in mass spectra reported in Figure 7 indicate that the impressive capability to resolve isobaric species is, nevertheless, limited by the space charge. Results of an attempt to experimentally evaluate ion coalescence in the 2xNADEL ICR cell are reported in Figure S9, Supporting Information. First, these results demonstrate that an ion coalescence threshold does exist for the approach implemented in this work. To quantitatively evaluate the envisioned benefits of ion detection at the true cyclotron frequency over the reduced cyclotron frequency, their coalescence thresholds are to be compared. However, that is a complicated task in regard to ensuring similar experimental procedures. Our current results compare data sets for ion detection at the reduced cyclotron frequency (1xNADEL ICR cell operated in the conventional FT-ICR regime) and ion detection at the true cyclotron frequency (2xNADEL ICR cell, with quadrupolar ion detection) acquired on the same 10 T LTQ FT instrument, Figure S9, Supporting Information. These results indicate that the true cyclotron frequency operation potentially increases the coalescence threshold 2-4-fold, thus extending the spectral dynamic range. These findings are in line with the previously reported conclusions of the computational experiments. ${ }^{39}$ 


\section{Conclusions}

The novel 2xNADEL ICR cells were proposed, designed, built, and successfully implemented on two commercial FT-ICR MS instruments from different manufacturers. The experimental results reported here demonstrate that these cells enable improved performance of FT-ICR MS at the true cyclotron frequency compared with the prior art. The improvements are particularly important for experiments with fluctuating ion sources (e.g., MALDI imaging), where ion abundance may vary over several orders of magnitude from scan to scan and from pixel to pixel.

Collectively, the results reported in prior literature and here reveal an intriguing and mostly unexplored nature of stable and coherent cyclotron motion of ions spatially dispersed in a large volume of an ICR cell, sustainable during the periods of up to minutes, in the non-uniform trapping electric fields. Owing to the extensive ion motion simulations performed for the NADEL ICR cells to date, ${ }^{38,} 39$ the described here dependencies and analytical characteristics of FT-ICR MS at the true cyclotron frequency could be rationalized by the current understanding of the corresponding ion motion and ion detection fundamentals. However, to comprehensively evaluate the potential practical benefits of this novel mode of ion motion, an optimization of experimental sequences of the employed FT-ICR MS instruments is needed. Besides, further developments of the underlying fundamentals are required to explore the reserves of performance improvement of the ICR cells that enable ion detection at the true cyclotron frequency. 


\section{Acknowledgments.}

We are grateful for financial support through the European Research Council (ERC Starting Grant 280271 to YOT) and the National FT-ICR network (FR 3624 CNRS). The research of OYT was supported by Peter the Great Saint Petersburg Polytechnic University in the framework of the Program "5-1002020”. We thank Ecole Polytechnique Fédérale de Lausanne for providing access to the $10 \mathrm{~T}$ LTQ FT instrument and Nicolas Elie for providing tissue samples for imaging experiments. The authors also express their gratitude to Thermo Fisher Scientific Inc. for providing access under license to LTQ FT transient signals and the advanced user interface, and to Bruker Daltonics Inc. for technical support on the ICR cell installation on SolariX XR.

\section{Supporting Information.}

The Supporting Information is available free of charge at

The Supporting Information contains nine figures. The SI figures provide details on the 2xNADEL ICR cells construction and their performance evaluation. The latter includes dependences of the observed $\omega_{c}$ and $\omega_{+}$ frequencies on the applied trapping potential and their SNR values on the excitation amplitude. Other examples include mass accuracy analyses for calibration mixtures and proteins, pixel-to-pixel ion signal intensity variation in MALDI imaging, the dependence of peak resolution and SNR on the detection period, including an example of ultra-high-resolution, and a spacecharge evaluation. 
Conflict of interest. The authors declare the following competing financial interest(s): A.K., K.N., and Y.T. are employees of Spectroswiss, which develops ICR cells. 


\section{References}

1. Scigelova, M., Hornshaw, M., Giannakopulos, A., Makarov, A.: Fourier Transform Mass Spectrometry. Molecular \& Cellular Proteomics. 10, M111.009431 (2011)

2. Ledford, E.B., Rempel, D.L., Gross, M.L.: Space charge effects in Fourier Transform Mass Spectrometry. I. Electrons. International Journal of Mass Spectrometry and Ion Processes. 55, 143-154 (1984)

3. Ledford, E.B., Rempel, D.L., Gross, M.L.: Space charge effects in Fourier transform mass spectrometry. II. Mass calibration. Analytical Chemistry. 56, 2744-2748 (1984)

4. Masselon, C., Tolmachev, A.V., Anderson, G.A., Harkewicz, R., Smith, R.D.: Mass measurement errors caused by "local" frequency perturbations in FTICR mass spectrometry. Journal of the American Society for Mass Spectrometry. 13, 99-106 (2002)

5. Wong, R.L., Amster, I.J.: Experimental evidence for space-charge effects between ions of the same mass-to-charge in Fourier-transform ion cyclotron resonance mass spectrometry. International Journal of Mass Spectrometry. 265, 99-105 (2007)

6. Affolter, M., Anderegg, F., Driscoll, C.F.: Space Charge Frequency Shifts of the Cyclotron Modes in Multi-Species Ion Plasmas. Journal of The American Society for Mass Spectrometry. 26, 330-336 (2015)

7. Aizikov, K., Mathur, R., O'Connor, P.B.: The spontaneous loss of coherence catastrophe in Fourier transform ion cyclotron resonance mass spectrometry. Journal of the American Society for Mass Spectrometry. 20, 247-256 (2009) 
8. Mitchell, D.W.: Theory of trapped ion motion in the non-quadrupolar electrostatic potential of a cubic ion cyclotron resonance cell. International Journal of Mass Spectrometry and Ion Processes. 142, 1$22(1995)$

9. Caravatti, P., Allemann, M.: The 'infinity cell': A new trapped-ion cell with radiofrequency covered trapping electrodes for Fourier transform ion cyclotron resonance mass spectrometry. Organic Mass Spectrometry. 26, 514-518 (1991)

10. Marshall, A.G., Chen, T.: 40 years of Fourier transform ion cyclotron resonance mass spectrometry. International Journal of Mass Spectrometry. 377, 410-420 (2015)

11. Marshall, A.G., Hendrickson, C.L., Jackson, G.S.: Fourier transform ion cyclotron resonance mass spectrometry: a primer. Mass spectrometry reviews. 17, 1-35 (1998)

12. Marshall, A.G.: Milestones in Fourier transform ion cyclotron resonance mass spectrometry technique development. International Journal of Mass Spectrometry. 200, 331-356 (2000)

13. Schaub, T.M., Hendrickson, C.L., Horning, S., Quinn, J.P., Senko, M.W., Marshall, A.G.: High-Performance Mass Spectrometry: Fourier Transform Ion Cyclotron Resonance at 14.5 Tesla. Analytical Chemistry. 80, 3985-3990 (2008)

14. Hendrickson, C.L., Quinn, J.P., Kaiser, N.K., Smith, D.F., Blakney, G.T., Chen, T., Marshall, A.G., Weisbrod, C.R., Beu, S.C.: 21 Tesla Fourier Transform Ion Cyclotron Resonance Mass Spectrometer: A National 
Resource for Ultrahigh Resolution Mass Analysis. Journal of The American Society for Mass Spectrometry. 26, 1626-1632 (2015)

15. Shaw, J.B., Lin, T.-Y., Leach, F.E., Tolmachev, A.V., Tolić, N., Robinson, E.W., Koppenaal, D.W., Paša-Tolić, L.: 21 Tesla Fourier Transform Ion Cyclotron Resonance Mass Spectrometer Greatly Expands Mass Spectrometry Toolbox. Journal of The American Society for Mass Spectrometry. 27, 1929-1936 (2016)

16. Hanson, C.D., Castro, M.E., Kerley, E.L., Russell, D.H.: Field-corrected ion cell for ion cyclotron resonance. Analytical Chemistry. 62, 520-526 (1990)

17. Guan, S., Marshall, A.G.: Ion traps for Fourier transform ion cyclotron resonance mass spectrometry: principles and design of geometric and electric configurations. International Journal of Mass Spectrometry and Ion Processes. 146-147, 261-296 (1995)

18. Boldin, I.A., Nikolaev, E.N.: Fourier transform ion cyclotron resonance cell with dynamic harmonization of the electric field in the whole volume by shaping of the excitation and detection electrode assembly. Rapid Communications in Mass Spectrometry. 25, 122-126 (2011)

19. Tolmachev, A.V., Robinson, E.W., Wu, S., Kang, H., Lourette, N.M., Paša-Tolić, L., Smith, R.D.: Trapped-Ion Cell with Improved DC Potential Harmonicity for FT-ICR MS. Journal of the American Society for Mass Spectrometry. 19, 586-597 (2008)

20. Kaiser, N.K., Savory, J.J., McKenna, A.M., Quinn, J.P., Hendrickson, C.L., Marshall, A.G.: Electrically compensated Fourier transform ion 
cyclotron resonance cell for complex mixture mass analysis. Anal Chem. 83, 6907-6910 (2011)

21. Misharin, A.S., Zubarev, R.A.: Coaxial multi-electrode cell ('O-trap') for high-sensitivity detection at a multiple frequency in Fourier transform ion cyclotron resonance mass spectrometry: main design and modeling results. Rapid Communications in Mass Spectrometry. 20, 3223-3228 (2006)

22. Nagornov, K.O., Gorshkov, M.V., Kozhinov, A.N., Tsybin, Y.O.: HighResolution Fourier Transform Ion Cyclotron Resonance Mass Spectrometry with Increased Throughput for Biomolecular Analysis. Analytical Chemistry. 86, 9020-9028 (2014)

23. Nagornov, K.O., Kozhinov, A.N., Tsybin, O.Y., Tsybin, Y.O.: Ion Trap with Narrow Aperture Detection Electrodes for Fourier Transform Ion Cyclotron Resonance Mass Spectrometry. Journal of The American Society for Mass Spectrometry. 26, 741-751 (2015)

24. Lozano, P., Catalina, D., Gavard, R., Arenas-Diaz, J.P., Thomas, M.J., Stranz, D.D., Mejía-Ospino, E., Guzman, A., Spencer, S.E.F., Rossell, D., Barrow, M.P.: Pushing the analytical limits: new insights into complex mixtures using mass spectra segments of constant ultrahigh resolving power. Chemical Science. 10, 6966-6978 (2019)

25. Smith, D.F., Kharchenko, A., Konijnenburg, M., Klinkert, I., Paša-Tolić, L., Heeren, R.A.: Advanced Mass Calibration and Visualization for FTICR Mass Spectrometry Imaging. Journal of The American Society for Mass Spectrometry. 23, 1865-1872 (2012) 
26. Bowman, A.P., Blakney, G.T., Hendrickson, C.L., Ellis, S.R., Heeren, R.M.A., Smith, D.F.: Ultra-High Mass Resolving Power, Mass Accuracy, and Dynamic Range MALDI Mass Spectrometry Imaging by 21-T FT-ICR MS. Analytical Chemistry. 92, 3133-3142 (2020)

27. Kooijman, P.C., Nagornov, K.O., Kozhinov, A.N., Kilgour, D.P.A., Tsybin, Y.O., Heeren, R.M.A., Ellis, S.R.: Increased throughput and ultra-high mass resolution in DESI FT-ICR MS imaging through new-generation external data acquisition system and advanced data processing approaches. Scientific Reports. 9, 8 (2019)

28. Park, S.-G., Anderson, G.A., Bruce, J.E.: Characterization of Harmonic Signal Acquisition with Parallel Dipole and Multipole Detectors. Journal of The American Society for Mass Spectrometry. 29, 1394-1402 (2018)

29. Kaiser, N., Quinn, J., Blakney, G., Hendrickson, C., Marshall, A.: A Novel 9.4 Tesla FTICR Mass Spectrometer with Improved Sensitivity, Mass Resolution, and Mass Range. Journal of The American Society for Mass Spectrometry. 22, 1343-1351 (2011)

30. Tolmachev, A.V., Robinson, E.W., Wu, S., Smith, R.D., Paša-Toli, L.: Trapping Radial Electric Field Optimization in Compensated FTICR Cells. Journal of The American Society for Mass Spectrometry. 22, 1334-1342 (2011)

31. Amster, I.J.: Fourier Transform Mass Spectrometry. Journal of Mass Spectrometry. 31, 1325-1337 (1996)

32. Gabrielse, G.: The true cyclotron frequency for particles and ions in a Penning trap. International Journal of Mass Spectrometry. 279, 107112 (2009) 
33. Ulmer, S., Blaum, K., Kracke, H., Mooser, A., Quint, W., Rodegheri, C.C., Walz, J.: Direct Measurement of the Free Cyclotron Frequency of a Single Particle in a Penning Trap. Physical Review Letters. 107, $103002(2011)$

34. Allemann, M., Kellerhals, H.P., Wanczek, K.P.: Sidebands in the ICR spectrum and their application for exact mass determination. Chemical Physics Letters. 84, 547-551 (1981)

35. Gabrielse, G.: Why is sideband mass spectrometry possible with ions in a Penning trap? Phys. Rev. Lett. 102, (2009)

36. Nagornov, K.O., Kozhinov, A.N., Tsybin, Y.O.: Fourier Transform Ion Cyclotron Resonance Mass Spectrometry at the Cyclotron Frequency. Journal of The American Society for Mass Spectrometry. 28, 768-780 (2017)

37. Miladinovic, S.M., Kozhinov, A.N., Tsybin, O.Y., Tsybin, Y.O.: Sidebands in Fourier transform ion cyclotron resonance mass spectra. International Journal of Mass Spectrometry. 325, 10-18 (2012)

38. Nagornov, K.O., Kozhinov, A.N., Tsybin, Y.O.: Cyclotron Phase-Coherent Ion Spatial Dispersion in a Non-Quadratic Trapping Potential is Responsible for FT-ICR MS at the Cyclotron Frequency. Journal of The American Society for Mass Spectrometry. 29, 63-77 (2018)

39. Driver, J.A., Nagornov, K.O., Kozhinov, A.N., Tsybin, Y.O., Kharchenko, A., Amster, I.J.: Multiparticle Simulations of Quadrupolar Ion Detection in an Ion Cyclotron Resonance Cell with Four Narrow Aperture Detection Electrodes. Journal of The American Society for Mass Spectrometry. 29, 51-62 (2018) 
40. Schweikhard, L., Lindinger, M., Kluge, H.J.: Quadrupole-detection FTICR mass spectrometry. International Journal of Mass Spectrometry and Ion Processes. 98, 25-33 (1990)

41. Heck, M., Blaum, K., Cakirli, R.B., Rodríguez, D., Schweikhard, L., Stahl, S., Ubieto-Diaz, M.: Dipolar and quadrupolar detection using an FT-ICR MS setup at the MPIK Heidelberg. Hyperfine Interact. 199, 347$355(2011)$

42. Touboul, D., Brunelle, A.: MALDI Mass Spectrometry Imaging of Lipids and Primary Metabolites on Rat Brain Sections. In: He L (ed.). Springer New York, New York, NY, (2015)

43. Kilgour, D.P.A., Wills, R., Qi, Y.L., O'Connor, P.B.: Autophaser: An Algorithm for Automated Generation of Absorption Mode Spectra for FTICR MS. Analytical Chemistry. 85, 3903-3911 (2013)

44. Zhurov, K.O., Kozhinov, A.N., Fornelli, L., Tsybin, Y.O.: Distinguishing Analyte from Noise Components in Mass Spectra of Complex Samples: Where to Cut the Noise? Analytical Chemistry. 86, 3308-3316 (2014)

45. Jertz, R., Friedrich, J., Kriete, C., Nikolaev, E.N., Baykut, G.: Tracking the Magnetron Motion in FT-ICR Mass Spectrometry. Journal of The American Society for Mass Spectrometry. 26, 1349-1366 (2015)

46. Schweikhard, L., Marshall, A.G.: Excitation modes for Fourier transform ion cyclotron resonance mass spectrometry. Journal of the American Society for Mass Spectrometry. 4, 433-452 (1993)

47. Palmblad, M., Håkansson, K., Håkansson, P., Feng, X., Cooper, H.J., Giannakopulos, A.E., Green, P.S., Derrick, P.J.: A 9.4 T Fourier Transform Ion Cyclotron Resonance Mass Spectrometer: Description 
and Performance. European Journal of Mass Spectrometry. 6, 267-275 (2000)

48. Tsybin, Y.O., Hendrickson, C.L., Beu, S.C., Marshall, A.G.: Impact of ion magnetron motion on electron capture dissociation Fourier transform ion cyclotron resonance mass spectrometry. International Journal of Mass Spectrometry. 255-256, 144-149 (2006)

49. Nikolaev, E.N., Jertz, R., Grigoryev, A., Baykut, G.: Fine Structure in Isotopic Peak Distributions Measured Using a Dynamically Harmonized Fourier Transform Ion Cyclotron Resonance Cell at 7 T. Analytical Chemistry. 84, 2275-2283 (2012)

50. Zubarev, R.A., Kruger, N.A., Fridriksson, E.K., Lewis, M.A., Horn, D.M., Carpenter, B.K., McLafferty, F.W.: Electron Capture Dissociation of Gaseous Multiply-Charged Proteins Is Favored at Disulfide Bonds and Other Sites of High Hydrogen Atom Affinity. Journal of the American Chemical Society. 121, 2857-2862 (1999)

51. Kozhinov, A.N., Zhurov, K.O., Tsybin, Y.O.: Iterative Method for Mass Spectra Recalibration via Empirical Estimation of the Mass Calibration Function for Fourier Transform Mass Spectrometry-Based Petroleomics. Analytical Chemistry. 85, 6437-6445 (2013)

52. Kilgour, D.P.A., Nagornov, K.O., Kozhinov, A.N., Zhurov, K.O., Tsybin, Y.O.: Producing absorption mode Fourier transform ion cyclotron resonance mass spectra with non-quadratic phase correction functions. Rapid Communications in Mass Spectrometry. 29, 1087-1093 (2015)

53. Gavard, R., Palacio Lozano, D.C., Guzman, A., Rossell, D., Spencer, S.E.F., Barrow, M.P.: Rhapso: Automatic Stitching of Mass Segments 
from Fourier Transform Ion Cyclotron Resonance Mass Spectra. Analytical Chemistry. 91, 15130-15137 (2019)

54. Catalina, D., Lozano, P., Thomas, M.J., Jones, H.E., Barrow, M.P.: Petroleomics: Tools, Challenges, and Developments. Annual Review of Analytical Chemistry. 13, null (2020)

55. Rodgers, R.P., Mapolelo, Mmilili M., Robbins, W.K., Chacón-Patiño, M.L., Putman, J.C., Niles, S.F., Rowland, S.M., Marshall, A.G.: Combating selective ionization in the high resolution mass spectral characterization of complex mixtures. Faraday Discussions. 218, 29-51 (2019)

56. Alexandrov, T., Ovchinnikova, K., Palmer, A., Kovalev, V., Tarasov, A., Stuart, L., Nigmetzianov, R., Fay, D., Gaudin, M., Lopez, C.G., Vetter, M., Swales, J., Bokhart, M., Kompauer, M., McKenzie, J., Rappez, L., Velickovic, D., Lavigne, R., Zhang, G., Thinagaran, D., Ruhland, E., Sans, M., Triana, S., Sammour, D.A., Aboulmagd, S., Bagger, C., Strittmatter, N., Rigopoulos, A., Gemperline, E., Joensen, A.M., Geier, B., Quiason, C., Weaver, E., Prasad, M., Balluff, B., Nagornov, K., Li, L., Linscheid, M., Hopf, C., Heintz, D., Liebeke, M., Spengler, B., Boughton, B., Janfelt, C., Sharma, K., Pineau, C., Anderton, C., Ellis, S., Becker, M., Pánczél, J., Violante, G.D., Muddiman, D., Goodwin, R., Eberlin, L., Takats, Z., Shahidi-Latham, S.: METASPACE: A community-populated knowledge base of spatial metabolomes in health and disease. bioRxiv. 539478 (2019) 
57. He, F., Hendrickson, C.L., Marshall, A.G.: Baseline Mass Resolution of Peptide Isobars: A Record for Molecular Mass Resolution. Analytical Chemistry. 73, 647-650 (2001) 


\section{Figure captions.}

Figure 1. Construction and operation of a 2xNADEL ICR cell. (Top left) design of the 2xNADEL ICR cell with four excitation grid-electrodes and four narrow aperture detection electrodes, containing trapping rings. (Top right) schematic representation of the 2xNADEL ICR cell with a quadrupolar ion detection circuit. Ion motion in the cell resulting in cyclotron frequency generation and detection is shown for 200 ions with an initial velocity spread defined before ion excitation. Snapshot of 2D projections of ion trajectories in the whole volume of the ICR cell at the detection period of $989 \mathrm{~ms}$ is shown in the plane perpendicular to the magnetic field axis. Ions were created with the predefined initial (pre-excitation) ion velocity $V_{z}: V_{z}=1.00+0.001^{*} \mathrm{n} \mathrm{mm} / \mathrm{us}$, where $\mathrm{n}=$ 0...200. (Bottom panel) SIMION-generated electric potential contours in the 2xNADEL ICR cell in the plane perpendicular to the magnetic field: (bottom left) the trapping potential distribution and (bottom right) the excitation potential contours with the adjacent electrodes wiring to the excitation amplifier. The employed simulation parameters: excitation electrodes at $-2 \mathrm{~V}$ and $2 \mathrm{~V}$ and trapping electrodes at $2 \mathrm{~V}$; all contours from $0.2 \mathrm{~V}$ to $2.0 \mathrm{~V}$ in 0.2 $\mathrm{V}$ increments.

Figure 2. The dependence of (top left) peak frequency and (bottom left) peak SNR on the applied trapping potential during ion detection. Experimental data were acquired in the (black) standard reduced cyclotron regime $\omega_{+}$with ParaCell (Bruker Daltonics) and (red) cyclotron frequency regime $\omega_{c}$ with 2xNADEL cell, both on a 9.4 T SolariX XR (Bruker Daltonics) instrument, 
using the isolated monoisotopic peak of doubly charged substance P at 674.47 $\mathrm{m} / \mathrm{z}$. The data at variable trapping potential were acquired with other fixed parameters (DC offsets, excitation power, etc.) tuned to the best performance of each ICR cell. Ion accumulation time was $200 \mathrm{~ms}$ for both data sets. The corresponding normalized frequency spectra are shown on the right panel.

Figure 3. Performance of $2 x N A D E L ~ I C R$ cell in calibration mixtures analysis. (Left panel) Single scan broadband mass spectrum of a mixture of polyphosphoric acid, poly- $\left(\mathrm{H}_{3} \mathrm{PO}_{4}\right)_{n}$, acquired in the cyclotron frequency regime using 9.4 T SolariX XR (Bruker Daltonics) equipped with 2xNADEL ICR cell. The mass error distributions for 11 monoisotopic peaks of poly-phosphoric acid were measured with external calibration in the mass range of $300-1500$ $\mathrm{m} / \mathrm{z}$ (middle panel) in 87 single scans using $9.4 \mathrm{~T}$ SolariX XR (Bruker Daltonics) and (right) in 2174 single scans using $10 \mathrm{~T}$ LTQ FT (Thermo Scientific).

Figure 4. Performance of $2 x N A D E L$ ICR cell for petroleomics-type applications. The shown example is an APPI petroleomics analysis using $10 \mathrm{~T}$ LTQ FT (Thermo Scientific) FT-ICR MS at the cyclotron frequency. (Left panel) averaged ( 21,000 scans) broadband mass spectrum of a crude oil fraction acquired using 2xNADEL ICR cell with the transient period of $3 \mathrm{~s}$ and processed in (black) magnitude (mFT) and (red) absorption (aFT, AutoVectis) modes. (Right panel) mass error vs. intensity plot calculated for 14'674 monoisotopic peaks identified in the aFT mass spectrum of the crude oil 
fraction. The total number of identifications for peaks above six standard deviations of noise, including heavy isotopologues, was 33'434.

Figure 5. Performance of $2 x N A D E L ~ I C R$ cell for imaging applications. $9.4 \mathrm{~T}$ SolariX XR (Bruker Daltonics) FT-ICR MS MALDI imaging experiment at the cyclotron frequency using 2xNADEL ICR cell: rat brain sample, 50 um spatial resolution, $T_{\text {acq }}=354 \mathrm{~ms}$, in total 50957 pixels. (Left panel) images of ions annotated in the MALDI FTMS dataset via METASPACE web-service. (Right panel) mass error distribution for corresponding annotated ions. Data was processed in aFT mode (AutoVectis) using fixed calibration coefficients (external calibration). Signal processing and visualization was carried out with Peak-by-Peak.

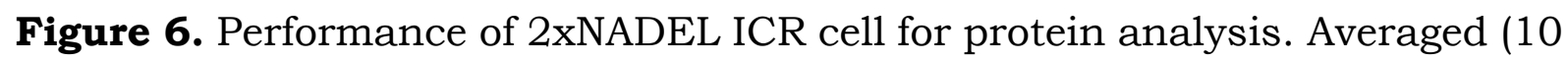
scans) broadband mass spectrum of myoglobin measured in the cyclotron frequency regime using 9.4 T SolariX XR (Bruker Daltonics) equipped with 2xNADEL ICR cell. Insets show a $5 \mathrm{~s}$ transient, isotopic envelope of the $18+$ charge state, and an example peak shape in (black) magnitude mode (mFT) and (red) absorption mode (aFT). The mass spectrum in aFT mode was generated using AutoVectis software.

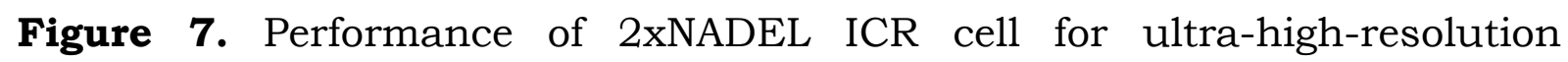
applications. Examples of ion motion coherence with a 9.4 T SolariX (Bruker Daltonics) equipped with 2xNADEL ICR cell. Shown are (left panel) an extended period (50 s) transient and corresponding (black) single and (red) 
averaged mass spectrum of a mixture of isobaric singly protonated peptides (heterodyne mode): EESTR, PMMSR, HDGHMR; and (right panel) an extended period (top) transient of $97 \mathrm{~s}$ and (bottom) corresponding single scan mass spectrum of a singly charged peptide (heterodyne mode). 
Figure 1.
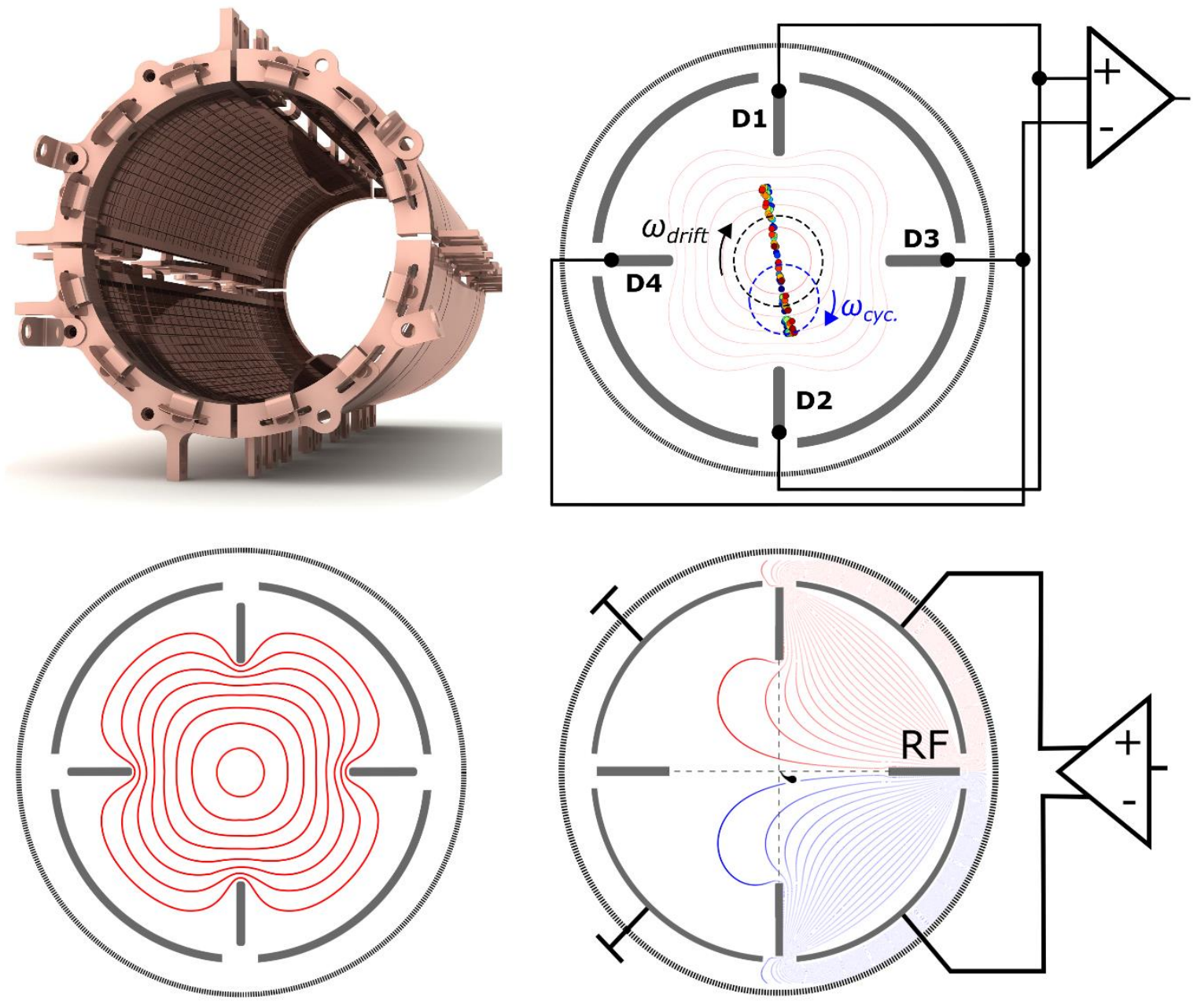
Figure 2.
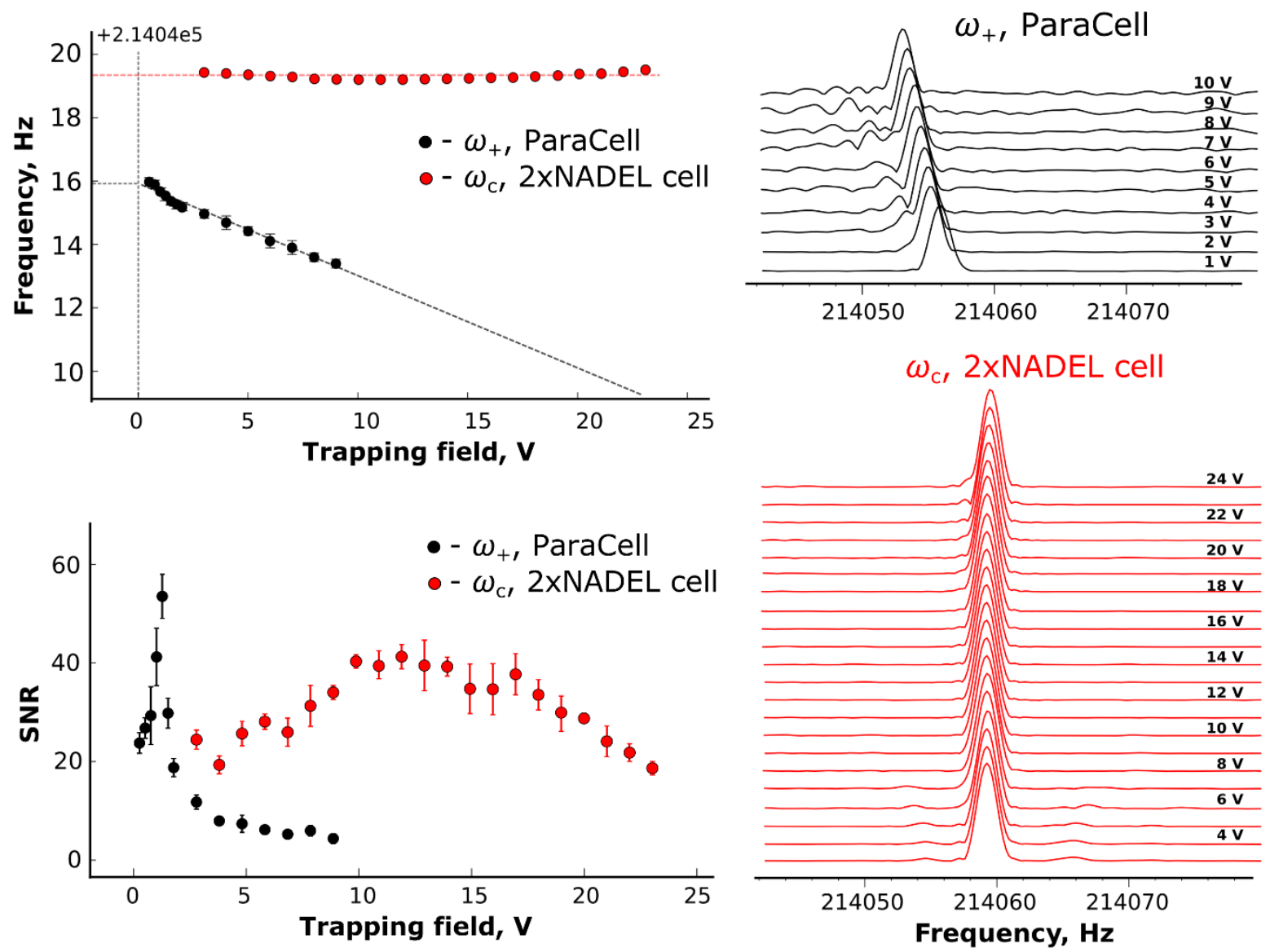
Figure 3.

9.4 T SolariX, $2 \times N A D E L$ cell, $\left(\mathrm{H}_{3} \mathrm{PO}_{4}\right)_{\mathrm{n}}$, single scan, $T_{\text {acq }}=1.5 \mathrm{~s}$

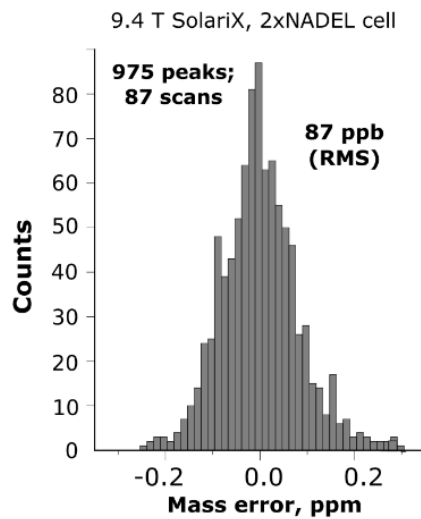

10 T LTQ-FT, 2XNADEL cell
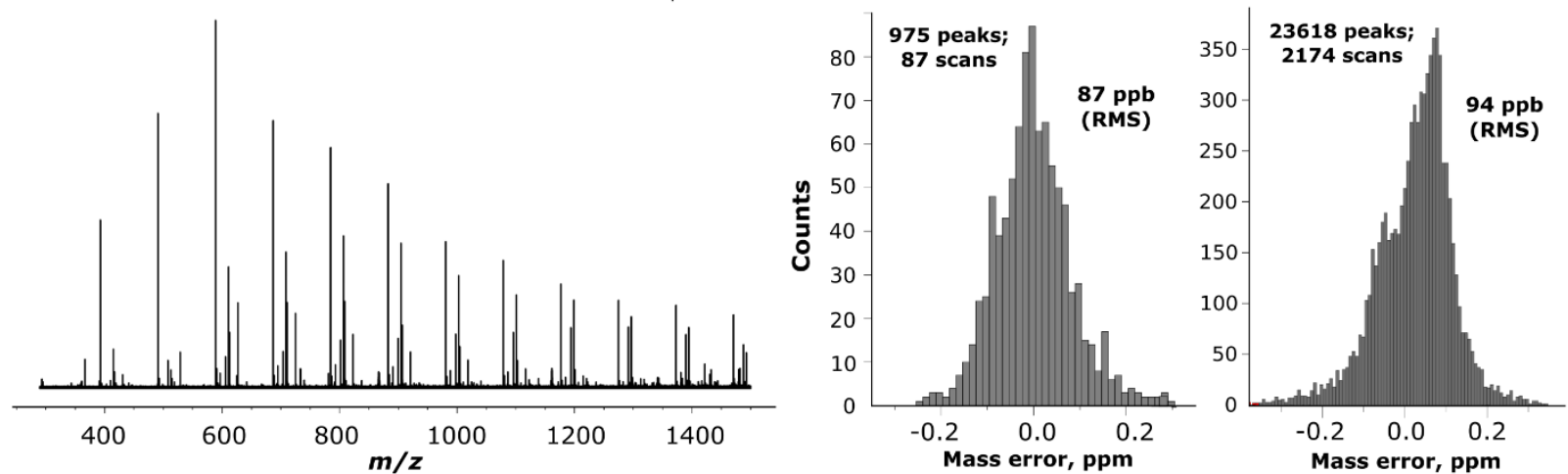
Figure 4.

10 T LTQ-FT (Thermo Scientific), 2xNADEL cell, crude oil fraction, $T_{\text {acq }}=3 \mathrm{~s}$

Npeaks: 14'674, RMS: 164 ppb
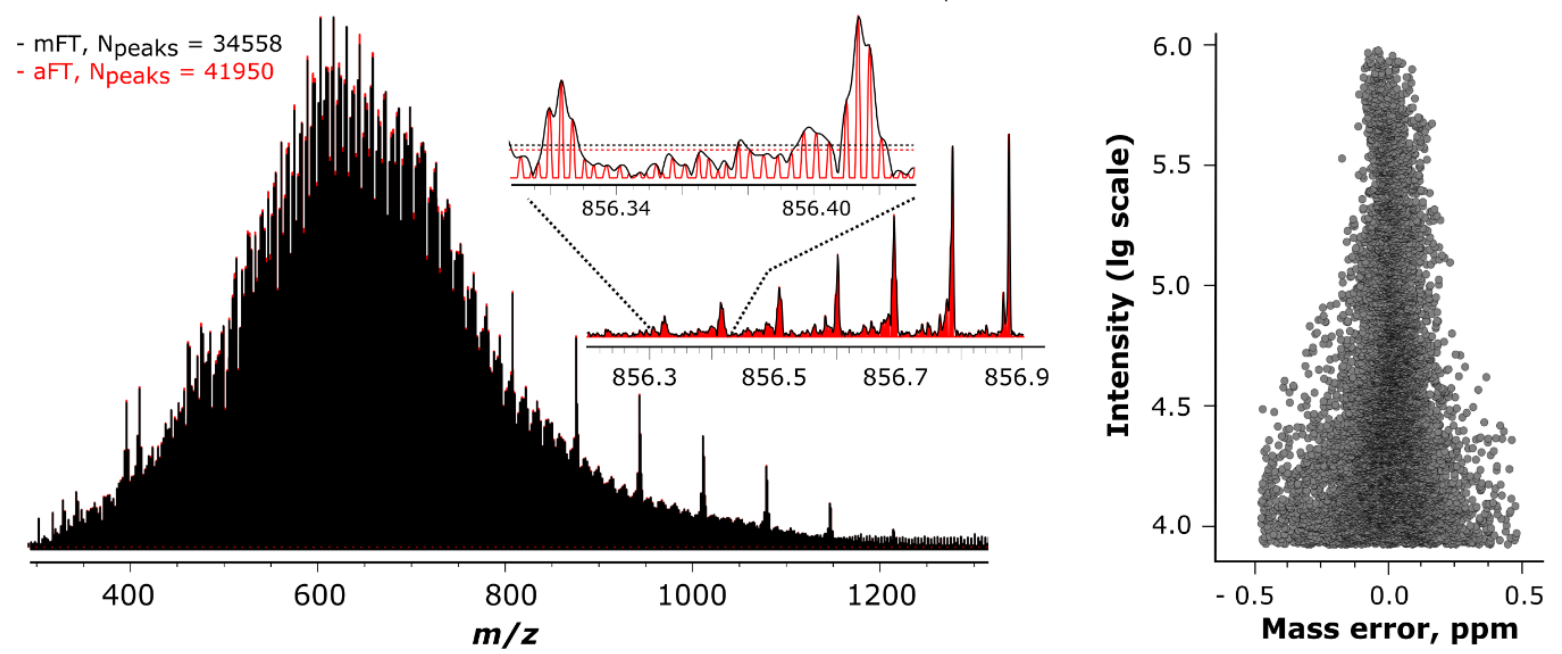
Figure 5.
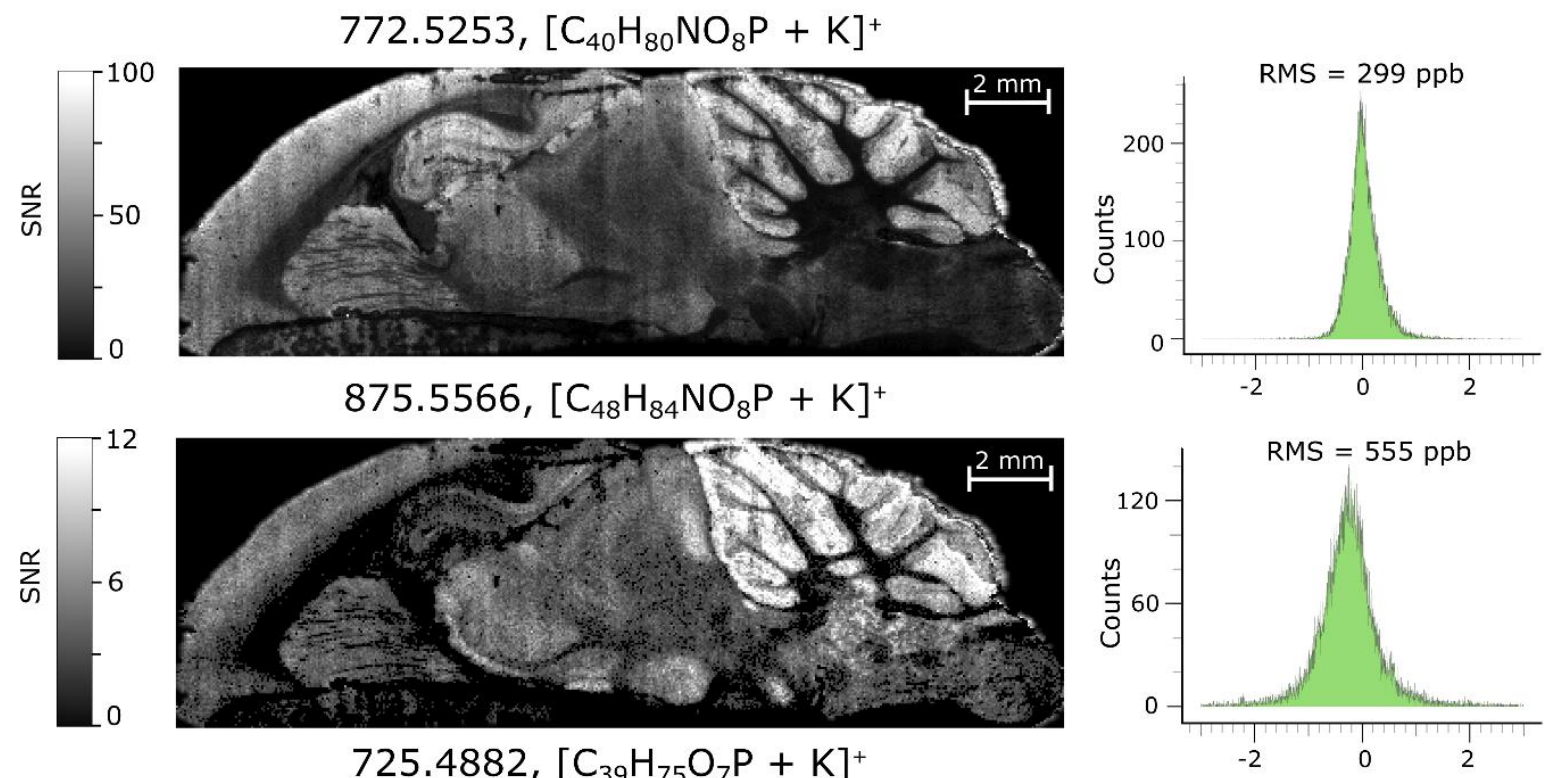

$\sum_{0}^{\alpha}{ }_{0.0}^{5.0}$

725.4882, $\left[\mathrm{C}_{39} \mathrm{H}_{75} \mathrm{O}_{7} \mathrm{P}+\mathrm{K}\right]^{+}$
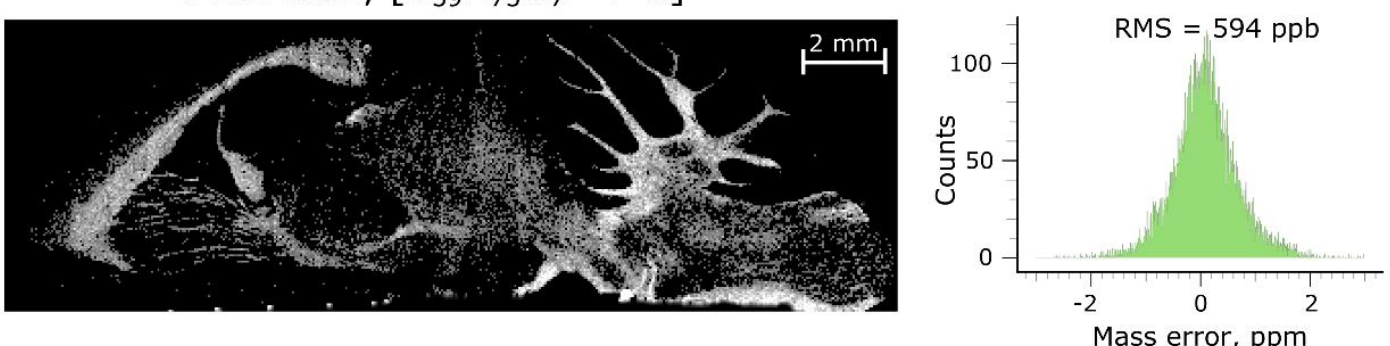
Figure 6.

9.4 T SolariX (Bruker Daltonics), 2xNADEL cell, myoglobin ( $17 \mathrm{kDa})$
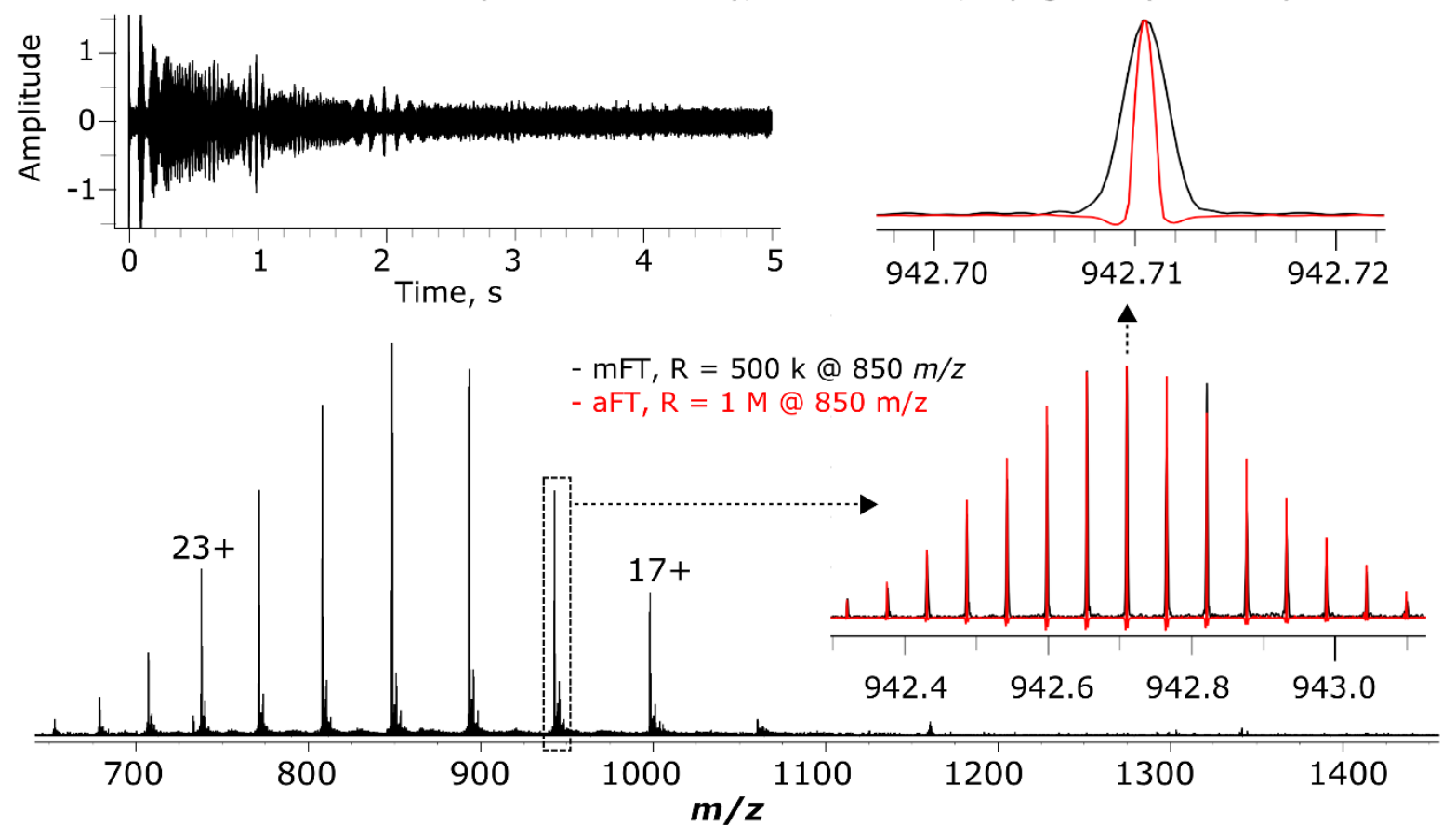
Figure 7.
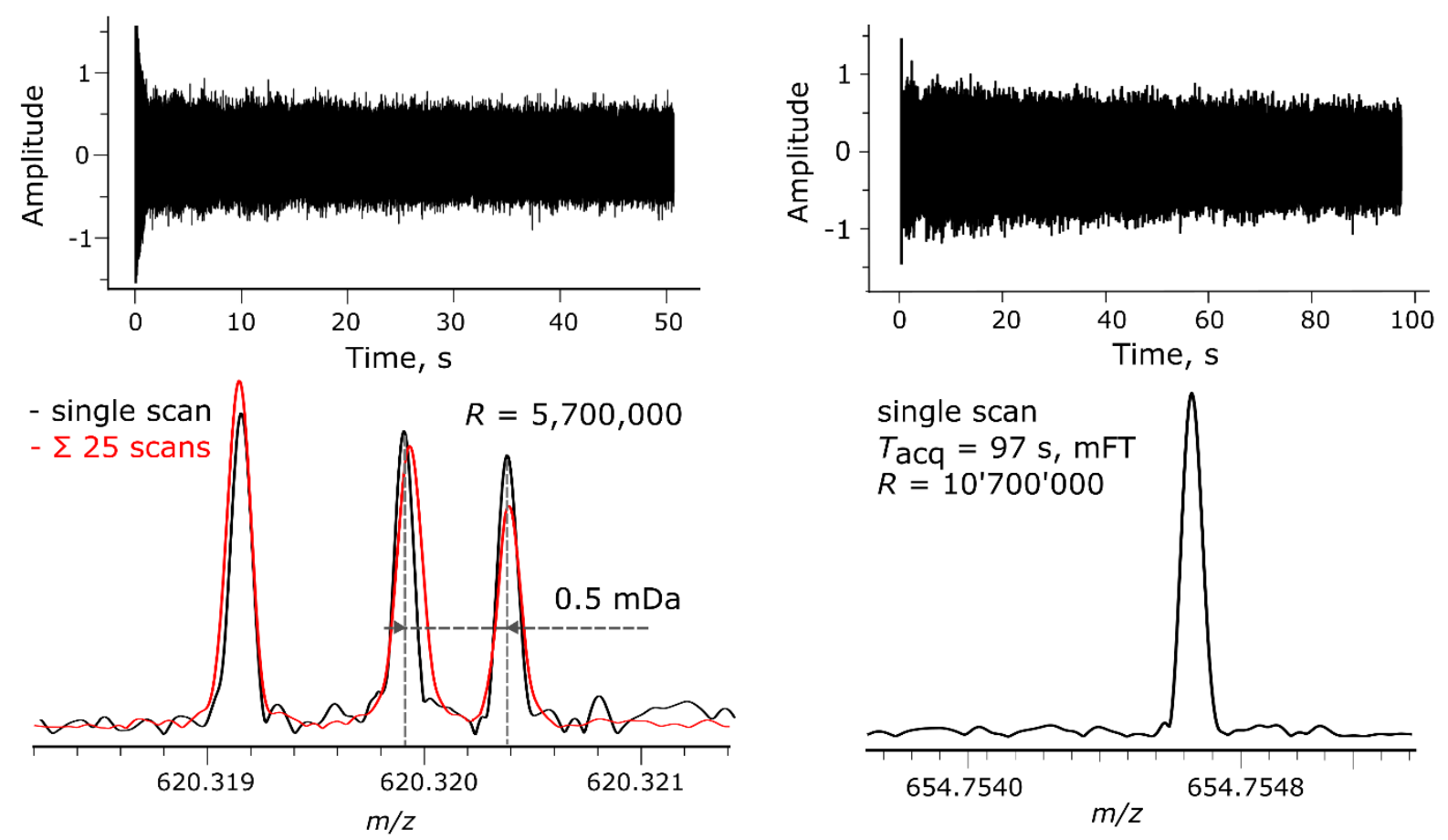


\section{For Table of Contents Use Only}

A narrow aperture detection electrodes ICR cell with quadrupolar ion detection for FT-ICR MS at the cyclotron frequency

Konstantin O. Nagornov, Anton N. Kozhinov, Edith Nicol, Oleg Yu. Tsybin, David Touboul, Alain Brunelle, and Yury O. Tsybin

Synopsis: We present a novel ICR cell, a 2xNADEL ICR cell, that employs quadrupolar ion detection to improve performance of FT-ICR MS at the true cyclotron frequency. The latter is characterized by relative independence of peak position on trapping fields, improving the corresponding analytical figures of merit and facilitating mass spectra calibration.
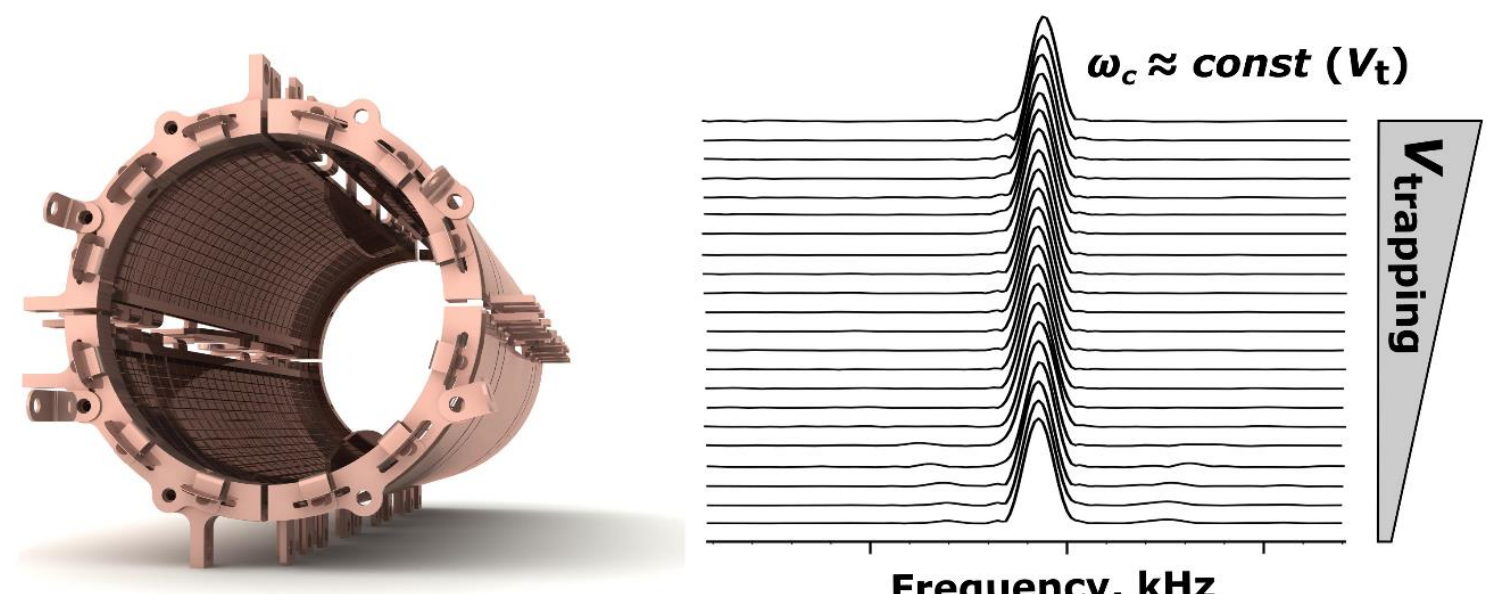

Frequency, kHz 